\title{
Estrategias instruccionales para promover el aprendizaje de la lectura musical en estudiantes de Educación Universitaria
}

\author{
Instructional strategies to promote the learning of musical reading in \\ University Education students
}

\author{
Ahiryn Andreina Yépez \\ ahirynyepez@gmail.com \\ Código ORCID: 0000-0003-0750-1396 \\ Universidad Pedagógica Experimental Libertador, Venezuela
}

- Artículo recibido en octubre 2019

- Arbitrado en noviembre 2019

\section{RESUMEN}

El presente estudio tuvo el propósito de presentar un procedimiento de investigación óptimo para proponer estrategias instruccionales destinadas a promover el aprendizaje de la lectura musical en estudiantes universitarios. Se formularon un conjunto de objetivos: a) Diagnosticar los conocimientos previos sobre lectura musical que poseen los estudiantes; b) diseñar un conjunto de estrategias instruccionales para promover el aprendizaje de la lectura musical y c) determinar la efectividad de la aplicación del conjunto de estrategias instruccionales para promover ese aprendizaje. Se diseñó como un Proyecto Especial que combinó estrategias de los enfoques cuantitativos y cualitativos. Se utilizaron como técnicas una encuesta de tipo dicotómica y la observación directa. Los resultados se sintetizaron en: a) el diagnóstico evidenció que los estudiantes poseían escasos conocimientos previos para la lectura musical; b) se logró diseñar un material con los requerimientos justos, y c) la aplicación de las estrategias diseñadas fueron efectivas para un aprendizaje significativo.

Palabras clave: Estrategias instruccionales; lectura musical; aprendizaje significativo, aprendizaje de la lectura musical

\section{ABSTRACT}

The purpose of this study was to present an optimal research procedure to propose instructional strategies aimed at promoting the learning of music reading in university students. A set of objectives were formulated: a) To diagnose the students' previous knowledge about musical reading; $b$ ) design a set of instructional strategies to promote the learning of musical reading and c) determine the effectiveness of the application of the set of instructional strategies to promote that learning. It was designed as a Special Project that combined strategies from the quantitative and qualitative approaches. A dichotomous survey and direct observation were used as techniques. The results were synthesized in: a) the diagnosis showed that the students had little prior knowledge for music reading; $b$ ) it was possible to design a material with the just requirements, and c) the application of the designed strategies were effective for meaningful learning.

Keywords: Instructional Strategies; music reading; meaningful learning, learning music reading 


\section{INTRODUCCIÓN}

El diseño de estrategias instruccionales para promover el aprendizaje de la lectura musical surgió de la necesidad de dar respuesta a una problemática encontrada en el curso de Lenguaje y Percepción Musical de la Especialidad Educación Musical del Instituto Pedagógico de Miranda de la UPEL. En su mayoría los estudiantes no poseen los conocimientos previos suficientes para poder alcanzar de manera efectiva los objetivos planteados en el curso, cuya línea de aprendizaje se fundamenta en la adquisición de competencias básicas que les permitan decodificar, leer e interpretar música escrita bajo diversas situaciones.

En función de lo trazado se propuso desarrollar, diseñar y aplicar un conjunto de estrategias instruccionales para promover el aprendizaje de la lectura musical en dichos estudiantes. Estas estrategias fueron validadas para ser aplicadas y posteriormente determinar su efectividad de acuerdo con el logro significativo de la adquisición de herramientas básicas que permitieron optimizar el aprendizaje de la lectura musical en el grupo de estudio (Yépez, 2013).

Desde un punto de vista educativo, la música puede ser concebida como un medio o como un fin en sí misma. Como medio, la educación musical permite motivar, desarrollar y reforzar nociones propias de otros aprendizajes, como fin constituye una excelente vía de expresión, comunicación y creación que ejercita la sensibilidad humana, la inteligencia creadora y la imaginación (Willems, 1981).

La educación musical forma de manera integral y globalizadora, proporciona experiencias cognitivas (lenguaje y ciencia) y sensitivas (arte) de un modo armónico aportando profundidad y una mayor capacidad de reflexión en aprendizajes futuros. En la Especialidad de Educación Musical de la Universidad Pedagógica Experimental Libertador, del Instituto Pedagógico de Miranda José Manuel Siso Martínez se crea el escenario perfecto para atender todos los procesos de enseñanza y aprendizaje con respecto al ámbito de la música. En esta especialidad se cubre la necesidad de formar profesionales de la docencia en esta área.

Uno de los aspectos relevantes establecidos en el Diseño Curricular (UPEL, 1996) señala que el perfil del egresado de la Especialidad en Educación Musical estará en la capacidad de: "Dominar la lectura musical en sus diferentes aspectos: rítmico, melódico y armónico a través del uso de la voz y de los instrumentos musicales" (p. 4). Por lo tanto, la lectura musical y el estudio del piano, la armonía, la técnica vocal y dirección coral, son de vital importancia para todo músico académico-universitario; por consiguiente, indiferentemente del 
contexto donde se desenvuelva debe ser capaz de dominar el lenguaje de la música, reconocida por la notación musical constituida por símbolos, términos $\mathrm{y}$ códigos utilizados $\mathrm{e}$ interpretados de la misma manera mundialmente.

Sin embargo, a la institución ingresan bachilleres sin estudios musicales previos quienes deben adquirir competencias básicas que van en función de la decodificación del lenguaje musical, en donde se hace necesario la discriminación auditiva de sonidos, el reconocimiento de las notas musicales (do, re, mi, fa, sol, la, si) en diversas claves (clave de sol, clave de fa o clave de do), el dominio de los signos de la música, figuras de notas (redondas, blancas, negras, corcheas, fusas, semifusas y sus diversas combinaciones) y silencios, reconocimiento de alteraciones (sostenido, bemol, becuadro), ejecución de diversos ritmos, entre otros. Estas competencias son fundamentales para una lectura óptima del lenguaje musical.

Los estudiantes revelan algunas debilidades con respecto a estas exigencias: dificultades en el reconocimiento del nombre de las notas en las líneas y espacios del pentagrama en clave de sol, deficiencias en la reproducción vocal de los sonidos, problemas de afinación (entendiendo por afinación como la acción de poner en correcta frecuencia los sonidos emitidos por la voz), dificultades en la identificación de figuras de notas, debilidades en la ejecución de ritmos, entre otros, lo que se reflejará en su desempeño real para la adquisición del aprendizaje de este curso.

En un diagnóstico previo se apreciaron factores concomitantes con esta situación: a) se observa que el grupo de estudiantes en formación docente es heterogéneo con respecto a los conocimientos previos sobre la lectura del lenguaje musical, lo que dificulta un diagnóstico general del curso; b) hay estudiantes con escaso conocimiento técnico sobre el lenguaje musical, pero que son grandes instrumentistas de oído con muchos años de experiencia; c) estudiantes que no tienen nociones ni técnicas, ni empíricas de la música; d) existen debilidades en el uso de los procesos mentales o cognitivos y esto trae como consecuencia un aprendizaje memorístico y poco significativo.

Por estas razones se hizo necesaria una investigación que aportara tanto a la comunidad estudiantil como a los docentes, estrategias que permitan superar estas debilidades y que a su vez logren consolidar la lectura musical en el nivel de pregrado. Al respecto se formularon las interrogantes: ¿Cuáles son los conocimientos musicales previos que poseen los estudiantes del curso Lenguaje y Percepción Musical del Instituto Pedagógico de Miranda José Manuel Siso Martínez?, ¿cuáles 
estrategias instruccionales permitirán promover el aprendizaje de la lectura musical en estos estudiantes? Y ¿cuán efectivas serán las estrategias instruccionales concebidas para el aprendizaje de la lectura musical?

Para dar respuestas a estas interrogantes, los propósitos de esta investigación fueron proponer $\mathrm{y}$ aplicar un conjunto de estrategias instruccionales para promover el aprendizaje de la lectura musical en estudiantes universitarios del Instituto Pedagógico de Miranda José Manuel Siso Martínez, previo al diagnóstico de sus conocimientos en el área.

El curso Lenguaje y Percepción Musical I es teórico-práctico y su objeto es que el estudiante adquiera las competencias necesarias para dominar efectivamente la lectura del lenguaje musical para hacer lecturas de líneas rítmico-melódicas a primera vista, logre tomar dictados rítmico-melódicos y pueda desarrollar habilidades más complejas asociadas a la lectura musical que le facilite el ensayo y montaje de grupos corales, bandas escolares, estudiantinas, entre otros, que se encuentren especialmente en el ámbito educativo musical. Con esta investigación se les ofreció estrategias y herramientas para codificar, almacenar y evocar información y así lograr un aprendizaje significativo de la lectura musical. Igualmente, a los docentes se les ha provisto de un recurso innovador para su mejor desempeño académico en el aula de clases, mediante un conjunto de estrategias instruccionales motivadores.

Sobre la materia, Furnó (2001) manifestó que la descripción de las actividades mentales complejas en el campo de la música encuentra en la psicología cognitiva, las neurociencias, la lingüística y la inteligencia artificial un campo fértil para su desarrollo, así como modelos teóricos y metodológicos permiten indagar la formación $\mathrm{y}$ adquisición de conceptos musicales a partir del análisis de factores intervinientes. Para estos casos, es determinante la formación de conceptos en general y la formación de conceptos musicales, en particular, conciliando procesos de atención, procesos de memoria y operaciones cognitivas sobre la información acústica retenida.

Según Castro (2003a) se entiende, en cuanto a la percepción y retención en la memoria de la altura tonal del lenguaje musical que los sujetos con mayor experiencia musical tendrán mayores probabilidades de analizar muestras musicales. Sin embargo, la intervención oportuna del docente durante la resolución de problemas puede resultar crucial para encaminar las respuestas de los estudiantes (Burcet y Herrera, 2008).

Más allá de estas apreciaciones, debe precisarse que la investigación se fundamentó principalmente en el enfoque cognitivo (Hernández, 2008), debido al énfasis en el estudio de los 
procesos internos que conducen el aprendizaje y la transformación que se opera en el individuo como "agente activo, cuyas acciones dependen en gran parte de las representaciones o procesos internos que él mismo elabora como producto de las relaciones previas con su entorno físico y social" (Poggioli, 1989). La información nueva, en consecuencia, se integra a la estructura cognoscitiva del individuo, se construyen nuevos significados y el material que aprende es potencialmente significativo para él (Gimeno y Pérez, 1998).

Ausubel (1976) distingue tres tipos de aprendizajes significativo, que van en función de la naturaleza de la nueva información y de su relación con las ideas activadas en la mente de la persona que aprende: el aprendizaje de representaciones, de conceptos y de proposiciones. Pero a medida que el individuo va recibiendo instrucción formal, se irá produciendo cada vez en mayor grado, una asimilación de conceptos, consistente en relacionar los nuevos conceptos con otros anteriormente formados (Pozo, 1989). La formación de conceptos en el aprendizaje de la lectura musical se encuentra vinculada a procesos de audio percepción, atención, desarrollo de la memoria auditiva, desarrollo de la memoria visual, retención de sonidos y clasificación del lenguaje de la música. Es allí donde el aprendizaje de representaciones previo a los conceptos, posterior a la formación de conceptos y el aprendizaje de proposiciones encuadra de manera perfecta en la consolidación de un aprendizaje significativo, ya que bajo esta estructura planteada en el enfoque cognitivo se logra profundizar en el aprendizaje de una manera secuencial y así garantizar que llegue la información de manera efectiva al estudiante.

Otro de los enfoques en el cual se fundamenta el estudio está referido al sociocultural, el cual se orienta bajo las ideas establecidas por Vigotsky (1978). Lo fundamental del enfoque consiste en considerar al individuo como el resultado del proceso histórico $\mathrm{y}$ social, donde el lenguaje desempeña un papel esencial. Para este autor, el conocimiento es un proceso de interacción entre el sujeto y el medio, pero el medio entendido social y culturalmente (Coll y Edwards, 1996).

Es por esta apreciación que se decide fundamentar bajo este segundo enfoque la investigación, en vista de que se piensa que algunas de las debilidades planteadas anteriormente referidas a las deficiencias que poseen algunos estudiantes del curso Lenguaje Percepción Musical, se adquirieron en el medio donde este se formó. Así mismo, sucede con el caso contrario, hay personas que son muy virtuosas en la ejecución e interpretación musical pero que no poseen estudios formales. Cada actividad realizada en la Educación Musical, tuvo una 
orientación con estos enfoques y las estrategias respondieron a una organización secuencial, por parte del docente, del contenido a aprender y la selección de los medios instruccionales idóneos (Smith y Ragan, como se citó en Alfonzo, 2003).

Como plantea (Flores, 2002), las estrategias de enseñanza se involucraron en la promoción de aprendizajes significativos a partir de los contenidos escolares; aquí el énfasis se pone en el diseño, programación, elaboración $\mathrm{y}$ realización de los contenidos a aprender por vía oral o escrita. Siguieron la línea según su momento de uso y presentación en: antes (Preeinstruccionales), durante (Coinstruccionales) o después (Postinstruccionales) de un contenido curricular específico (Díaz y Hernández, 2002) dependiendo de la naturaleza de esta disciplina.

La lectura del lenguaje musical comprende el conocimiento y dominio de una serie de elementos (ritmo, melodía y armonía), signos (pentagrama, clave de sol, clave de fa en 3era y 4ta línea, clave de do en 2da, 3era y 4ta línea, líneas divisorias de compás, etc.), figuras de notas (redonda, blanca, negra, corchea, semicorchea, fusa, semifusa), alteraciones (sostenido, doble sostenido, bemol, doble bemol y becuadro), entre otros que le permiten al individuo hacer un reconocimiento visual auditivo de un fragmento musical planteado hasta lograr interpretarlo, bien sea de manera vocal o a través de un instrumento.
López (2004) expresa que la lectura musical implica abrir una partitura y sin ayuda de un instrumento saber cómo suena, tal como ocurre en la lectura literaria.

Otros aspectos de gran relevancia que permiten una buena lectura musical se traduce cuando el participante puede: desarrollar y lograr una óptima audición interna de los sonidos, así mismo apropiarse de una efectiva lectura musical le permitirá asumir compromisos en cuanto a la conducción de grupos musicales, vocales u orquestales. Acceder a la literatura musical de todos los tiempos, transcribir su música, así como también, transcribir música de otros.

Para elaborar el diseño instruccional se tomaron como punto de partida las bases psicológicas de los elementos fundamentales de la música, según Willems (1984): el ritmo, la melodía y la armonía. De los elementos del ritmo (Castro, 2003b; Ortiz, 1997; Pérez, 2000; Reyes, 1986; Rodríguez, 2005 y Vasconcelos; 1986) se privilegiaron: el pulso; el acento; las figuras de notación rítmica que representan la duración del sonido, "sin embargo solo se presenta una duración finita o fija, en tiempo real cuando se relaciona con un pulso y tiempo específico" (Gauldi, 2009, p. 36); las figuras de silencio; el compás y su cifra indicadora; compases simples y compases compuestos; relación entre cada figura musical, la cifra numérica 
que representa; las líneas divisorias o barra de compás se utilizan para separar unos compases de otros.

También se tomaron en cuenta las unidades de la melodía (Castro, 2003b; Córdoba, 2005; Fradera, 2009): el pentagrama y sus líneas adicionales; las claves, de fa, de sol y de do; la historia del nombre de las notas; las alteraciones (Rodríguez, 2003): el sostenido, el becuadro y bemoles. También existe el doble sostenido y el doblo bemol, con cada una de sus funciones; la Escala General; las escalas diatónicas, constituidas por tonos y semitonos tanto diatónicos como cromáticos.

Se precisaron los elementos de la armonía, especialmente algunos detalles sobre la formación y enlace de los acordes, donde se regula la concordancia entre sonidos que suenan de manera simultánea y su enlace con sonidos vecinos o próximos (Córdoba, 2005; Manrique, 2010) y la tonalidad, un grupo de notas relacionadas con un centro común o tónica sistema (Cantore, 2008).

No se dejaron de lado para la elaboración del diseño instruccional los mecanismos relacionados con la audición (Betés, 2000; Fernández, 2005) y la especificidad del oído musical del que se suele hablar de tres tipos de oído: el oído interno, el oído relativo y el absoluto. En los tres casos la memoria y la carga afectiva son fundamentales. Además se estudió lo propio del oído rítmico, el melódico y armónico.

\section{MÉTODO}

De acuerdo con las características de la investigación, se centró en un modelo mixto con los enfoques cualitativo y cuantitativo. Hernández, Fernández y Baptista (2006) manifiestan que en el modelo mixto, ambos enfoques se combinan en la mayoría de las etapas del proceso de investigación. Se utilizaron las técnicas del enfoque cuantitativo para diagnosticar los conocimientos previos que poseían los estudiantes del curso Lenguaje y Percepción Musical I y posteriormente se utilizó la metodología cualitativa para describir e interpretar las características y actuación de los participantes en la aplicación de las estrategias diseñadas.

El diseño obedece a la modalidad de Proyecto Especial el cual, según la UPEL (2006) consiste en "trabajos que lleven a creaciones tangibles, susceptibles de ser utilizadas como soluciones a problemas demostrados" (p. 17). Es así que las estrategias instruccionales se diseñaron en un material escrito dirigido a los estudiantes del curso. Las actividades se llevaron a cabo en una investigación de campo para indagar la incidencia y valores en que se manifiesta una o más variables de estudio correspondiente a un grupo de personas u objetos. La investigación de Campo es definida por UPEL (2006) como: 
El análisis sistemático de problemas en la realidad, con el propósito bien sea de describirlos, interpretarlos, entender su naturaleza y factores constituyentes, explicar sus causas y efectos, o predecir su ocurrencia, haciendo uso de métodos característicos de cualquiera de los paradigmas o enfoques de investigación conocidos o en desarrollo. Los datos de interés son recogidos en forma directa de la realidad (p. 18).

La información fue recogida en el aula de clases de Educación Musical con un carácter descriptivo, por cuanto se "busca especificar las propiedades, características y los perfiles de personas, grupos, comunidades, procesos, objetivos o cualquier otro fenómeno que se someta a un análisis" (Hernández, Fernández y Baptista, 2006, p. 102).

Con ello se describió cómo fue el comportamiento y el desenvolvimiento de los estudiantes del curso Lenguaje y Percepción Musical I al aplicarles en diversas sesiones las estrategias instruccionales para promover de manera significativa el aprendizaje y competencias adquiridas en la lectura musical.

Los constructos de mayor consistencia para su análisis fueron las estrategias de aprendizaje y los conocimientos previos que poseen los estudiantes en lectura musical, vistos como conductas de entrada. El sistema de aprendizaje se estableció a través de encuentros en varias modalidades, con los estudiantes: presencial semanal, presencial quincenal y a distancia; en esta última el participante es responsable de su propio aprendizaje, el docente desempeña un rol de orientador y facilitador de los contenidos a aprender (Flores, 200).

Los sujetos de investigación fueron catorce estudiantes del curso Lenguaje y Percepción Musical I de la carrera Educación Musical en el Instituto Pedagógico de Miranda José Manuel Siso Martínez. Inicialmente, el grupo fue heterogéneo en cuanto a sus conocimientos y estudios previos a su ingreso al instituto: seis bachilleres con estudios musicales avanzados, dos bachilleres expertos en el dominio de un instrumento con estudios de un conservatorio (pianistas, cantantes, guitarristas, entre otros), cuatro bachilleres con conocimientos de música pero sin estudios formales $\mathrm{y}$ dos bachilleres sin ningún conocimiento técnico del lenguaje musical.

El ambiente fue un salón de música con características específicas para la especialidad Educación Musical: una pizarra acrílica con pentagrama, ocho teclados, un órgano, dos estantes (uno para libros de la especialidad y otro para los instrumentos pequeños tales como: cuatro, mandolina, instrumentos de percusión menor, redoblantes, entre otros), sillas, mesas y un archivo con diversas partituras. 
Para la recolección de datos se acudió a "los procedimientos y actividades que permiten al investigador obtener la información necesaria para dar respuesta a su pregunta de investigación" (Hurtado, 1998, p. 409) con instrumentos de recolección como "recursos metodológicos que materializan la obtención de los datos, informaciones $\mathrm{y} / \mathrm{o}$ aspectos relevantes" (Flames, 2001, p. 27). Las técnicas empleadas fueron la encuesta y la observación y los instrumentos fueron una prueba escrita, una prueba diagnóstica práctica, el diario del profesor y un autoinforme.

La encuesta fue aplicada al inicio del período académico; este diagnóstico se efectuó en dos sesiones de clases: en la primera sesión se aplicó la prueba escrita y en la segunda, la prueba diagnóstica práctica. La prueba escrita es una diagnosis dividida en dos partes: reconocimiento de signos musicales con su significado e identificación de términos musicales igualmente con su significado. La prueba diagnóstica práctica "Conocimientos Previos para La Lectura Musical" fue diseñada y aplicada por la autora del estudio. E1 instrumento consta de 17 ítems distribuidos en dos aspectos: oído rítmico y oído melódico; se diseñó para ser respondida con una escala de ejecución. El propósito de este instrumento fue determinar las conductas de entrada de los estudiantes del curso Lenguaje y Percepción Musical I, con respecto a los signos, símbolos, términos y códigos necesarios para lograr la decodificación en la lectura musical.

Durante la aplicación de las estrategias instruccionales diseñadas, se reportó información relevante de lo ocurrido durante siete sesiones de clases en el diario del profesor. Asimismo, los autoinformes fueron aplicados al final de cada clase para que los estudiantes proporcionaran información escrita sobre sí mismos y sobre distintos aspectos como: las decisiones que tomaron para resolver las tareas, qué les pareció fácil y cuáles fueron las dificultades que tuvieron que superar, entre otros. Como apoyo y guía se les ofrecieron una lista de interrogantes que podían responder libremente: ¿Comprendí qué son los elementos de la música? Explica las decisiones tomadas para realizar el ejercicio sobre los elementos de la música. ¿Qué tanto me estoy esforzando para comprender los elementos de la música? ¿Cuáles puntos se me han hecho fáciles? ¿Qué puntos no me han quedado claros? ¿Qué he aprendido del tema? ¿Cómo lo aprendí? ¿Qué me falta por aprender? ¿Qué me falló y cómo puedo solucionarlo? ¿Me gusta la actividad referente a cada uno de los elementos de la música? Explique

La prueba práctica fue sometida a un proceso de validez y confiabilidad (García, 2002). La confiabilidad de este instrumento resultó de su aplicación a un grupo piloto perteneciente a la 
especialidad Educación Musical, y su inmediata revisión. En tanto que su validez se obtuvo por medio de juicio de expertos realizado por 3 profesionales: un magister en estrategias de aprendizaje y dos especialistas en el área de Música, quienes administran los cursos Lenguaje y Percepción Musical I y II. La validez y la confiabilidad permitieron hacer ajustes definitivos al instrumento. Esto confirmó su aplicabilidad.

\section{Fases de la Investigación}

Las tres fases de trabajo fueron: fase I, diagnóstico (prueba escrita y prueba práctica, que determinaron los conocimientos previos de los estudiantes). La fase II, referida al Diseño de las Estrategias Instruccionales, elaboradas en función de las debilidades presentadas por los participantes en la fase I y la fase III, referida a la aplicación de cada una de las estrategias previamente diseñadas.

En primera instancia todos los estudiantes debían demostrar sus conocimientos reconociendo y reproduciendo rítmicamente $\mathrm{o}$ vocalmente aspectos asociados al oído rítmico, al oído melódico y al oído armónico. Igualmente, debían reproducir corporalmente los patrones rítmicos presentados. Cada ítem a evaluar estaba acompañado por una escala de estimación representada en números del uno al cinco, donde el número uno expresaba la menor frecuencia y el cinco la mayor. Se valoró la ejecución del participante y se registró en la escala de ejecución. Para la segunda parte de este instrumento, se ubicaron ocho aspectos melódicos, donde el participante debía realizar la discriminación auditiva y reproducción vocal de sonidos, presentados por el evaluador con el apoyo del teclado. Al igual que la primera parte cada ítem a evaluar estaba acompañado por una escala.

Finalmente, para la tercera y última parte del instrumento, se establecieron cuatro aspectos armónicos, donde el estudiante debía identificar y reproducir vocalmente grupos de sonidos simultáneos, así como realizar improvisaciones bajo una estructura tonal determinada. La evaluación se llevó a cabo bajo el mismo procedimiento de las anteriores partes.

E1 segundo instrumento estuvo estructurado en dos partes, cuya intención era determinar en qué medida los estudiantes conocían el lenguaje formal de la música representada en signos y términos. En la primera parte, luego de establecer las instrucciones, se colocaron dos columnas en donde debían marcar con una "X" presencia o ausencia del rasgo (Sí o No). De aquellos que identificaran, se les solicitaba que expresara en una o varias palabras su significado o para qué se utilizaba en el lenguaje musical.

La segunda parte de la prueba teórica estaba asociada a la identificación de términos técnicos del 
lenguaje musical. Así mismo, se presentó una columna que contenía una lista de cincuenta y dos términos utilizados en el lenguaje de la música para marcar positivo o negativo sobre su conocimiento. El instrumento fue aplicado por un especialista en el área y se tomaron una jornada de clases en cada momento de aproximadamente dos horas para llevar a cabo las evaluaciones.

En la fase II, se diseñaron las estrategias instruccionales que fueron aplicadas al curso Lenguaje y Percepción Musical I con el desarrollo de siete estrategias para dar respuestas a las debilidades que arrojaron los participantes en las pruebas diagnósticas realizadas en la fase I.

Estas estrategias fueron estructuradas en siete clases tipo taller con apoyo de un material instruccional escrito. Cada estrategia no solo fue orientada en función de las debilidades presentadas por los estudiantes, sino también en función de los contenidos que deben ser consolidados en este curso. Su aplicación fue durante todo el período académico, tiempo estimado para lograr el tercer objetivo de la investigación. Las clases tipo taller se desarrollaron con la intención de proveer a los estudiantes de estrategias que le permitieran lograr la identificación, decodificación e interpretación efectiva de la lectura musical, siendo este el objetivo primordial del curso.
Las actividades para cada sesión estuvieron estructuradas de la siguiente manera: a) una introducción donde se les presentaba un material que le permitía preparar a la audiencia sobre el contenido que se desarrollaría en cada sesión, con esto se lograba llamar su atención, motivarlos y activar los conocimientos previos, seguidamente se establecían cuáles eran los objetivos que se trabajarían para cada lección; b) durante el desarrollo se les presentó el material instruccional, se establecieron los conceptos con sus respectivos ejemplos, se respondieron preguntas y se aclararon las dudas; c) seguidamente se procedía a desarrollar una serie de ejercicios propuestos en el material instruccional escrito que iban en función de cada lección, en este aspecto se incorporaron unos ejercicios denominados de Profundización, que invitan a una mayor reflexión por parte del estudiante y que permite desarrollar aspectos más complejos asociados a cada una de las lecciones; d) se establecían las conclusiones de la lección vista, y e) se les hacía entrega de un autoinforme que debían responder, con la intención de que ellos mismos valoraran cómo había sido su actuación en relación con la clase.

Todas las estrategias tipo taller estuvieron apoyadas en un material instruccional escrito diseñado por la autora de esta investigación. A este material se le denominó: "Estrategias Instruccionales para el curso Lenguaje 
y Percepción Musical I". El enfoque de este material teórico-práctico estuvo orientado a apoyar y consolidar los procesos cognitivos requeridos para que el estudiante de música pueda adquirir las competencias, habilidades y destrezas necesarias en la práctica de una lectura musical efectiva.

La fase III se dedicó a la aplicación de las estrategias para promover el aprendizaje de la lectura musical se procedió a aplicarlas. Se recolectó la información durante las sesiones, con el "Diario del Profesor" y el "Autoinforme".

\section{RESULTADOS}

Para el análisis de esta primera fase referida al diagnóstico de los conocimientos previos que poseen los estudiantes del curso de Lenguaje y Percepción Musical I, se procedió a tabular los resultados de la siguiente manera: Se desarrolló el análisis de la escala, por medio de una tabla donde se presenta la frecuencia de incidencia del grupo que se registró en cada uno de los aspectos evaluados. En la Tabla 1 se muestran los resultados de la prueba diagnóstica práctica donde los números reflejan una valoración de la ejecución del participante, expresados de la siguiente manera: el número uno (1) representa que Nunca realizó la ejecución, el número dos (2) muestra una ejecución que Casi Nunca la realizó, el número tres (3) expresa una ejecución que Algunas veces se presentó, el número cuatro (4) una ejecución que Casi Siempre se presentó y el número cinco (5) una ejecución que Siempre se realizó. En el instrumento, se evaluó del oído rítmico, seguidamente del oído melódico y para finalizar el oído armónico (Tabla 1). Allí

Tabla 1.Frecuencia grupal por cada uno de los indicadores de la Prueba Diagnóstica Práctica

\begin{tabular}{|c|c|c|c|c|c|c|}
\hline Ítem & OÍDO RÍTMICO & 1 & 2 & 3 & 4 & 5 \\
\hline 1 & $\begin{array}{l}\text { Reconoce y reproduce patrones rítmicos utilizando } \\
\text { como figuras de notas blancas, negras, corcheas, } \\
\text { semicorcheas y sus respectivos silencios. }\end{array}$ & & & 1 & 1 & 12 \\
\hline 2 & $\begin{array}{l}\text { Reproduce patrones rítmicos con presencia de } \\
\text { saltillos y tresillos simples }\end{array}$ & & & 3 & 1 & 10 \\
\hline 3 & $\begin{array}{l}\text { Reproduce ritmos combinados con las dos manos y } \\
\text { la voz }\end{array}$ & & 2 & 3 & 4 & 6 \\
\hline 4 & $\begin{array}{l}\text { Reproduce ritmos combinados llevando en una } \\
\text { mano el pulso y en la otra el ritmo }\end{array}$ & & 3 & 2 & 4 & 5 \\
\hline 5 & $\begin{array}{l}\text { Reproduce birritmias corporales con manos, pie y } \\
\text { voz }\end{array}$ & & 5 & 5 & 1 & 3 \\
\hline
\end{tabular}




\begin{tabular}{|c|c|c|c|c|c|c|}
\hline Ítem & OÍDO MELÓDICO & 1 & 2 & 3 & 4 & 5 \\
\hline 6 & Discrimina diferencias de altura & & & 11 & 2 & 11 \\
\hline 7 & Reproduce vocalmente intervalos & & & 4 & 4 & 6 \\
\hline 8 & $\begin{array}{l}\text { Discrimina auditivamente diferentes tipos de } \\
\text { intervalos }\end{array}$ & & & 3 & 5 & 6 \\
\hline 9 & Reproduce vocalmente escalas & & & 1 & 3 & 10 \\
\hline 10 & Reproduce vocalmente arpegios & & & 2 & 4 & 8 \\
\hline 11 & Reproduce vocalmente cromatismos & & & 8 & 2 & 4 \\
\hline 12 & $\begin{array}{l}\text { Discrimina auditivamente diferencias entre escala, } \\
\text { arpegio y cromatismo }\end{array}$ & & & 2 & 3 & 9 \\
\hline 13 & Reproduce ejercicios rítmico-melódicos & & & 6 & 5 & 2 \\
\hline Ítem & OÍDO ARMÓNICO & 1 & 2 & 3 & 4 & 5 \\
\hline 14 & Identifica auditivamente acordes y tonalidades. & & 2 & 4 & 1 & 7 \\
\hline 15 & $\begin{array}{l}\text { Reproduce vocalmente cada uno de los sonidos que } \\
\text { forman un acorde. }\end{array}$ & & & 4 & 4 & 6 \\
\hline 16 & Improvisa melodías sobre una base armónica. & & & 1 & 7 & 6 \\
\hline 17 & $\begin{array}{l}\text { Canta una melodía conocida sobre una base tonal } \\
\text { determinada. }\end{array}$ & & & 4 & 4 & 6 \\
\hline
\end{tabular}

Fuente: Elaboración propia.

Según la prueba diagnóstica referida al ritmo, se observa en el grupo debilidades marcadas, cuando se les solicita realizar ritmos combinados con las dos manos y la voz, cuando se les pedía reproducir ritmos combinados llevando en una mano el pulso y en otra el ritmo, así como cuando se les invitaba a realizar birritmias corporales con manos, pie y voz. Todo esto indica que el trabajo rítmico-corporal fue necesario incorporarlo en el planteamiento de las estrategias instruccionales diseñadas.

En referencia a la evaluación sobre el oído melódico la estadística realizada demuestra marcadas debilidades principalmente en la reproducción rítmico-melódica, al parecer al grupo entrevistado se le dificultaba escuchar, retener y reproducir patrones rítmicomelódicos. Otro de los aspectos que demuestran fallas es la reproducción vocal de cromatismos y en una menor proporción la discriminación y reproducción de intervalos. Los resultados arrojados en este aspecto precisaron la importancia de incorporar en el diseño de las estrategias principalmente ejercicios rítmicomelódicos que le permitan al estudiante lograr un mejor audio percepción y desarrollo de su memoria auditiva.

En relación con el último aspecto evaluado sobre el oído armónico, el estudio demuestra que los participantes tienen mayores dificultades al reproducir vocalmente cada uno de los sonidos que formen un acorde; en menor proporción también presentan algunas debilidades al momento de improvisar melodías sobre una base armónica.

En el segundo instrumento se mostraba a los estudiantes quince signos musicales fundamentales para precisar la frecuencia de reconocimiento, con las opciones SÍ o NO. Los resultados demostraron que los estudiantes en la 
mayoría de los casos logran reconocer algunos signos comúnmente utilizados en el lenguaje de la música; sin embargo, se presenta una constante generalizada en esta población de estudio, referida al poco conocimiento en referencia al significado o definición técnica que corresponde a los elementos presentados en esta primera parte de la prueba diagnóstica teórica.

En la segunda parte del instrumento, se esperaba, igualmente que reconocieran los términos musicales, con las opciones SÍ o NO. Se presentaban los términos vinculados al ritmo: compás, síncopa y contratiempo, tresillo; términos asociados al elemento melódico: escala dominante, tónica, sensible y mediante, la escala y a la armadura de llave; términos asociados al elemento armónico: tetracorde y arpegio, aumentado, disminuido, disonancia y consonancia, tritono; términos asociados a la dinámica en la música, a saber: Fraseo, Matices, Acento y el Staccato. Los resultados mostraron que los estudiantes lograron reconocer con facilidad $\mathrm{y}$ fluidez términos como: Pentagrama, Redonda, Metrónomo, Ligadura, Claves, Diatónico, Da Capo. Sin embargo al momento de expresar su definición pocos logran detallar su significado.

Estas pruebas permitieron valorar dónde se encontraban las debilidades para esta población, lo que a su vez permitió proyectar la organización de las estrategias idóneas que ayuden a transformar las debilidades en fortalezas por parte de los estudiantes.

La siguiente fase se dedicó a diseñar el material a partir de los resultados precedentes con sus respectivas estrategias y lecciones. En el material se incluyeron siete lecciones denominadas de la siguiente manera: Lección $\mathrm{N}^{\circ} 1$. El Sonido. Materia prima de la Música. Lección $\mathrm{N}^{\mathrm{o}}$ 2. Elementos de la Música. Lección $\mathrm{N}^{\circ}$ 3. Las Figuras de Notas. Lección $\mathrm{N}^{\mathrm{o}}$ 4. Las Notas Musicales. Lección $\mathrm{N}^{\mathrm{o}}$ 5. Ritmos Simples $\mathrm{y}$ Combinados. Lección $\mathrm{N}^{\mathrm{o}} 6$. Alteraciones. Tono y Semitono y Lección $N^{\circ}$ 7. La Tonalidad Mayor. Se elaboró un diseño uniforme con la firme intención de que se pudiera visualizar fácilmente la información, también se incorporaron al final de cada lección actividades prácticas tanto escritas como dinámicas como es el caso de la percusión corporal, así como también la utilización de la voz. La estructura a nivel global del material permitió que a pesar del lenguaje técnico fuese de fácil acceso y entendimiento para los participantes.

El material educativo diseñado fue, inmediatamente, validado por varios expertos: dos especialistas en contenido y uno en diseño instruccional. El material se compuso de: portada, índice del contenido, introducción donde se establece su estructura, concepto y fundamentación teórica. La validación por expertos arrojó una calificación establecida entre 0 y 1 de $0,96 \%$. El 
material se diseñó en función de lo que plantea el enfoque constructivista, ya que los estudiantes deben lograr los objetivos previamente planteados, realizando el procesamiento de la información de manera constructiva, es decir, activando sus conocimientos previos en apoyo a la lectura integrándola con la nueva información planteada en el material.

$\mathrm{El}$ diseño de este material tuvo como finalidad sustancial que los estudiantes: a) Pudieran acceder a la información a través de un conjunto de estrategias que facilitaran su aprendizaje, b) lograran la decodificación efectiva y rápida del lenguaje musical, y c) transfieran el conocimiento de la lectura musical para poder arribar a niveles más profundos de la teoría musical.

De seguida se procedió a su aplicación con lo que se pudo alcanzar logros significativos en la población de la especialidad Educación Musical. Esos logros van desde el aprendizaje de ritmos combinados utilizando las dos manos y la realización de birritmias corporales (manos, pie y voz). Igualmente, luego de las sesiones de clase los estudiantes pudieron reproducir patrones rítmicomelódicos y llevar a cabo la reproducción vocal de cromatismos, así como intervalos. Finalmente, los estudiantes lograron reproducir vocalmente los sonidos de un acorde e improvisar melodías sobre una base armónica, hasta reconocer significados y definiciones técnicas de los elementos musicales.
Es preciso destacar que los logros de este estudio permitieron afirmar que se han alcanzado las competencias prescritas en el Diseño Curricular de la Universidad Pedagógica Experimental Libertador (UPEL, 1996) donde se manifiesta que el objetivo fundamental de la especialidad es formar profesionales docentes en Educación Musical que estén capacitados para: a) Orientar el conocimiento y desarrollo de las potencialidades musicales, $b$ ) estimular y desarrollar la creatividad y la actuación espontánea del grupo y del individuo, utilizando la música como medio de expresión, c) organizar y dirigir agrupaciones vocales, instrumentales y mixtas, d) elaborar arreglos musicales para agrupaciones vocales, instrumentales y mixtas, e) desarrollar la cultura y la sensibilidad musical, f) promover los valores culturales del país, la región y la comunidad en la cual se desempeña, g) Orientar en el conocimiento y valoración de otras culturas, a través de la música, relacionándola con las demás manifestaciones del arte, $h$ ) investigar y desarrollar, a partir de su práctica pedagógica innovaciones y procedimientos en el campo de la enseñanza musical, i) orientar el desarrollo de hábitos de trabajo en el campo musical, j) Actualizar a los docentes, músicos en ejercicio, en el conocimiento especializado de la pedagogía musical. 


\section{CONCLUSIONES}

El trabajo por etapas realizado en esta investigación permitió dar respuesta a todos los objetivos planteados. En primer término se logró un diagnóstico profundo de la realidad de los estudiantes de educación musical. Esto fue posible gracias a una exploración de los conocimientos de lectura musical desde la teoría y desde la práctica a partir de dos instrumentos previamente validados. El segundo objetivo de esta investigación estuvo relacionado al conjunto de estrategias diseñadas para promover el aprendizaje de la lectura musical, las cuales se ajustaron positivamente a las debilidades presentadas por los estudiantes en el diagnóstico. Fue determinante la forma de organizar los contenidos de las estrategias en función de: activar los conocimientos previos de los estudiantes, posteriormente desarrollar la teoría con ejemplos sustanciales, puntualizar las ideas relevantes y finalmente desarrollar las actividades teórico-prácticas sugeridas para cada lección. Es importante destacar que fue de gran ayuda conocer en detalle las debilidades arrojadas por los estudiantes, lo que a su vez permitió diseñar las estrategias idóneas a las necesidades presentadas por el grupo de estudio.

En referencia al tercer objetivo de esta investigación sobre la aplicación de las estrategias diseñadas para promover el aprendizaje de la lectura musical, sirvió para que los estudiantes realizaran una autovaloración de cómo estaban realizando cada una de las actividades en pro de su aprendizaje significativo. Los procesos de construcción del conocimiento por parte de los estudiantes se vieron altamente influenciados por el análisis, la comparación y la clasificación tanto visual como auditiva, a lo que debían involucrarse de manera activa para lograr con éxito la transferencia del conocimiento. En la aplicación, un aspecto fundamental fue el de plantear las actividades de manera prácticas y colectivas, ya que generaban un avance importante de manera grupal $e$ individual. Muestra de ello fueron los ejercicios rítmico-corporales que se realizaron al inicio de la aplicación de las estrategias referida a las figuras de notas ello permitió que los estudiantes aprendieran con rapidez la clasificación y nombre de las notas por medio del trabajo corporal.

\section{REFERENCIAS}

Alfonzo, A. (2003). Estrategias instruccionales. Recuperado de http://www.medusa.unimet.edu.ve/ educacion/fbqi21/estrategias.pdf

Ausubel, D. (1976). Psicología educativa. México: Trillas

Betés, M. (2000). Fundamentos de Musicoterapia. Barcelona, España: Morata

Burcet, M. y Herrera, R. (2008). El rol de la lectoescritura musical en etapas iniciales de la formación audioperceptiva. Un estudio desarrollado a partir de la experiencia de estudiantes no 
videntes. Argentina: Facultad de Bellas Artes de la Universidad Nacional de La Plata

Castro, E. (2003a). La actividad cerebral en la percepción y retención en la memoria de la altura tonal del lenguaje y la música. México: Universidad Nacional Autónoma de México

Castro, M. (2003b). Música para todos. Una introducción al estudio de la música. Costa Rica: Universidad de Costa Rica

Coll, C. y Edwars, D. (1996). Enseñanza, aprendizaje y discurso en el aula: aproximaciones al estudio del discurso educacional. Madrid, España: Fundación Infancia y Aprendizaje

Córdoba, C. (2005). Diccionario de Música. España: Universal

Díaz Barriga, F. y Hernández, G. (2002). Estrategias docentes para un Aprendizaje significativo. Una visión Constructivista. México: Mc GrawHill

Fernández, A. (2005). Así se habla: nociones generales de fonética general y española. Madrid, España: Horsori

Flames, A. (2001). Como colaborar en un trabajo de grado de enfoque cualitativo para optar a los títulos de: Técnico Superior, Universitario o equivalente a Magíster o Doctorado. Calabozo, Guárico: Abel Flames

Flores, C. A. (2002). Estrategias Instruccionales para la comprensión de la lectura en estudiantes de Educación Integral en el Instituto Pedagógico de Miranda José Manuel Siso Martínez. (Tesis de maestría).Universidad Pedagógica Experimental Libertador, Miranda, Venezuela

Furnó, L. (2001). La formación de conceptos musicales. Estudio desarrollado en el marco del Programa de Incentivos al docente Investigador. Argentina: Facultad de Bellas Artes de la Universidad Nacional de La Plata

García, F. (2002). El cuestionario. Recomendaciones metodológicas para el diseño de un cuestionario. Argentina: Limusa/ Noriega Editores

Gimeno, P. y Pérez, R. (1998). Comprender y transformar la enseñanza. México: Morata

Hernández, G. (2008). Paradigmas en psicología de la educación. México: Paidós

Hernández, R., Fernández, C. y Baptista, P. (2006). Metodología de la Investigación. México: McGrawHill Interamericana

Hurtado, J. (1998). Metodología de la Investigación Holística. Caracas: Fundación Sypal

López, C. (2004). Lectura Musical. E1 Ritmo y la Melodía. Recuperado de http://www.musicaviva.com.ar/blo g/2007/11/24

Ortiz, M. (1997). Música, Educación y Desarrollo. Caracas: Monte Ávila

Pérez, M. (2000). Diccionario de música y los Músicos I. España: Istmo

Poggioli, L. (1989). Estrategias cognitivas: una revisión teórica y empírica. En Puerta, A. y otros. Psicología Cognoscitiva. Desarrollo y perspectiva. Madrid: McGraw-Hill

Poggioli, L. (2007). Estrategias de adquisición de conocimiento. Caracas: Polar

Pozo, I. (1989). Teorías cognitivas del aprendizaje. México: Morata

Reyes, A. (1986). Prolegómenos a la Teoría Literaria. México: Fondo de Cultura Económica

Rodríguez, A. (2003). Música I. Madrid, España: Editex 
Rodríguez, A. (2005). Música y Educación secundaria obligatoria. Madrid, España: Editex

UPEL. (1996). Diseño Curricular de la Especialidad Educación Musical. Caracas: Vicerrectorado de Docencia UPEL. (2006). Manual de Trabajos de Grado de Especialización y Maestría y Tesis Doctorales. Caracas: FEDUPEL

Vasconcelos, J. (1986). Una teoría del ritmo, el monismo estético. Recuperado de http//:www.cialc.unam.mx/pensam ientoycultura/biblioteca/virtual/dicc ionario/ritmo/htm

Willems, E. (1981). El valor humano de la Educación Musical. Barcelona, España: Paidós

Willems, E. (1984). Bases psicológicas de la educación musical. Barcelona, España: Paidós

Yépez, A. (2013). Estrategias instruccionales para promover el aprendizaje de la lectura musical en estudiantes de Educación Universitaria en el Instituto Pedagógico de Miranda José Manuel Siso Martínez. (Trabajo de Maestría). Instituto Pedagógico de Miranda, Universidad Pedagógica Experimental Libertador, Miranda, Venezuela 